\title{
THE INFLUENCE OF THE SURFACE TENSION OF THE CULTURE MEDIUM ON THE GROWTH OF BACTERIA.**
}

\author{
By W. P. Larson, W. F. Cantwell and T. B. Hartzell.
}

(From the Department of Bacteriology and Immunology, Unirorsity of Minnesota, Minnesota.)

$\mathrm{T}^{\mathrm{T}}$ HERE is probably no phase of bacteriologic research that has occupied the attention of investigators more than the preparation of culture mediums. Most of such investigations have been directed toward. supplying bacteria with the nutritive material best adapted to their specific individual recuirements. Aside from its reaction, the physico-chemical state of the medium has received but little attention.

It is the purpose of this paper to record the behavior of some of the common bacteria when cultivated on medium of low surface tension.

Our investigation naturally began with a search for suitable substances with which to lower the surface tension of mediums. It was soon found that substances that would lower the surface tension of water would not always act satisfactorily in lowering the tension of broth or other mediums. Furthermore, only such surface tension depressants could be used as were of themselves toxic neither to bacteria nor to laboratory animals into which cultures or filtrates were subsequently to be injected. It was found that soap fulfilled our requirements better than anything else tried. A large variety of commercial soaps were used, after first being purified and neutralized. They all possessed certain disadvantages, however, as they invariably caused a marked turbidity of the medium. Furthermore, the

\footnotetext{
*Aided by a Grant from the National Dental Research Association.
}

surface tension of the medium depressed by the various soaps would not remain constant, due to the fact that soap tends to hydrolyze when diluted, which causes the tension to rise. Studies were therefore made in an effort to secure a soap that would be perfectly clear when ir solution, and which would be sufficiently stable to prevent the objectionable fluctuations in the surface tension of the medium. It was found that pure castor oil saponified with $\mathrm{NaOH}$ met these requirements.

A number of neutralized fatty acids form a clear solution when hot, but become turbid and viscous on cooling. Solutions of castor oil soap on the other hand remain perfectly clear when cooled, do not hydrolyze on sterilization in flowing steam, nor do they become viscous when cooled.

Castor oil soap is prepared by adding an alcoholic solution of $\mathrm{NaOH}$ to castor oil slightly in excess of its saponification equivalent. The mixture is then heated over the water-bath until saponification is complete when the soap is dissolved in hot absolute alcohol to which is added a few drops of phenolphthalein. Hydrochloric acid, diluted with alcohol, is then added drop by drop until the soap solution is neutral. It is then filtered on paper to remove the chlorids. The excess of alcohol may then be removed by evaporation, after. which the soap solution is poured into a saturated solution of $\mathrm{NaCl}$ to remove the glycerol and other impurities. After further filtration followed by washing with a saturated solution of sodium chlorid the soap is ready for use. 
The drop method was used in measuring the surface tension.

Weight of number drops of nedium

Weight of number drops of water

In order to give a general idea of the comparative surface tension of various fluids it may be stated that that of water is 73 , alcohol 22 , and ether 16 dynes.

The broth prepared in our laboratory by standard methods has a surface tension of about 59 dynes. This may vary slightly, but it was always found to be between 58 and 60 dynes, 59 being the value usually obtained. The surface tension of broth may be lowered to any desired point down to 32 dynes by adding the necessary quantity of soap solution to the medium.

In growing bacteria on broth of lower surface tension we were impressed by the behavior of the $B$. subtilis when the tension had been depressed below 45 dynes. When grown on such medium this organism no longer forms a pellicle, but grows down in the body of the medium. When the tension of the broth is depressed to below 45 dynes the growth of the B. subtilis is largely at the bottom of the tube. It was further observed that spore formation was markedly suppressed and sometimes entirely absent, especially after cultivation on low tension mediums for several generations. B. subtilis grown on medium of low surface tension may sometimes be rendered sterile by heating to 60 degrees for an hour which indicates that spore formation has been entirely suppressed. It is generally supposed that $\mathrm{B}$. subtilis grows on the surface of broth because of its great demand for oxygen.

Since our work has shown that this organism is capable of growing thruout the medium, even at the bottom of the tube, it is evident that its oxygen requirement is not the chief reason for surface growth on ordinary broth. In this connection we would not overlook the fact that lowering the surface tension of water increases its ability to dissolve oxygen and other gases. A striking feature common to most bacteria which grow on the surface of liquid mediums, particularly B. subtilis, B. mesentericus, and $B$. tuberculosis and others, is that whenever there is pellicle formation there is little or no growth except at the very surface. In inoculating a flask of glycerol broth with B. tuberculosis large numbers of organisms may sink to the bottom of the flask, but only those that remain at the surface show signs of multiplying. Other bacteria, such as B. coli, the cholera spirillum, first show signs of development at or near the surface even tho there is no pellicle formation. What can be the explanation of this selective site of so many bacteria? It is possible that the surface of the medium certain substances are concentrated which favor, or are even necessary, for the metabolism of many microorganisms. It is a well known fact that surface energy tends to reduce itself to a minimum, and that this may be accomplished in two ways: (1) by the reduction of the surface area of the liquid, or (2) by reducing the surface tension, or both. The surface tension of the liquid is reduced to the lowest point possible thru the concentration of the surface tension depressants at the surface of the liquid. B. subtilis and other bacteria, which show such predilection for the surface of the medium, probably select this zone because the surface tension reducing substance concentrated there are required for their metabolism. The pellicle formation is probably a mere coincident, the surface tension of the fluid being sufficient to support the weight of the organisms just as certain insects may be supported on the surface of water brcause of its high surface tension.

The question naturally suggests itself as to whether or not all aerobic bacteria might not become pellicle formers were the surface tension of the culture medium sufficiently high. Since it is impossible to raise the tension of a me- 
dium much above that of water, this experiment has its limitations. However, we have found that B. coli as well as some of the paratyphoid bacteria form well developed pellicles when grown on an inorganic medium having a surface tension of 72.2 dynes.

Investigations have also conducted with reference to the influence of the surface tension of the medium on some of the ccmmon anaerobic bacteria, such as B. tetani, B. botulinus, B. chauvei. It was found that $B$. tetani grew well under ordinary aerobic conditions proving the surface tension of the medium had been properly depressed. B. tetani produces a potent toxin when grown on ordinary broth, the surface tension of which has been reduced to about 40 dynes.

This would seem to indicate that we cannot draw a sharp line between the aerobic and anaerobic bacteria altho there can be no question but that some bacteria demand a liberal supply of oxygen, while others cannot thrive where the supply is too liberal. In this connection it may be pointed out that one of th: yopular methods of producing anaerobinsis is to cover the medium with a layer of sterile oil, the object of the oil buing to exclude oxygen from the medium which it covers.

Since cil is a much better solvent of oxygen than water, is it reasonable to suppose that oil actually does exclude oxygen? If the tube were sealed off immediately after introducing oil into the test tube it might be argued that the oil, because of its being a better oxygen solvent than the medium, would cause the gas to diffuse from the medium into the orl Jater; but since the sealing of the ube following the introduction of the oil is not necessary to effect the socalled anaerobic condition, this is obviously not the explanation. A little experiment which shows that the oil does not exclude oxygen from culture medium may be made by inoculating a tube of broth with a culture of B. subtilis after which the inoculated medium may be covered with a layer of sterile oil; 48 hours following this inoculation it will be found that the subtilis has formed a very heavy pellicle just beneath the oil layar, showing that oxygen does pass from the oil into the medium, otherwise this organism would not be able to grow at this point. The oil undoubtedly favors the growth of anaerobic bacteria because it reduces the surface tension of the medium, and as a result the surface tension depressants contained in the medium are not concentrated at the surface as would ordinarily be the case, but are dispersed thruout the medium where they can serve the metabolic requirements of the anaerobic bacteria that under ordinary circumstances would be able to avail themselves of these surface tension lowering substances because of the excess of oxygen at the surface where they are concentrated.

The mere act of lowering the surface tension of the medium will not cause all anaerobes to grow under ordinary aerobic conditions. Such a germ as B. chauvei fails to show any appreciable development under such conditions.

Most bacteria seem to grow best when the surface tension has not been depressed below 53 to 55 dynes, altho most of the more common bacteria, such as the staphylococcus, B. coli, B. typhosus, and B. paratyphosus show some growth when the surface tension has been depressed as low as 32 dynes.

Surface tension below 45 dynes tends to cause many bacteria to agglutinate, thus giving the impression that the growth is entirely at the bottom of the test tube this being particularly true of the typhoid and paratyphoid bacilli. The streptococci and pneumocecci do not grow well where the surface tension has been brought below 50 dynes. The pneumococci show a tendency to disintegrate when grown on a medium of a low surface tension. This observation suggests that the solution of pneumococci by bile may be due, in part at least, 
to the lowering of the surface tension which bile induces. B. tuberculosis grows very well on medium of low surface tension. Like $B$. subtilis it no longer grows with pellicle formation, but grows at the bottom of the medium.

The pathogenicity of bacteria seems to be profoundly affected by the surface tension of the medium on which they are grown. We hope to give a detailed report on the relation of pathogenicity and toxin formation of bacteria to the surface tension of the medium in a subsequent article.

\section{SUMmaRY.}

The growth of bacteria on ordinary broth is greatly influenced by the surface tension of the medium.
A sodium soap of castor oil proved to be a practical surface tension depressant.

All pellicle formers cease to grow at the surface where the tension is below 45 dynes.

The tindency of B. subtilis to form spores is considerably reduced when the organism is grown on broth medium of low surface tension.

Some anaerobes, B. tetani in particular, grow well under ordinary aerobic conditions on medium of reduced surface tension. The layer of oil often used to cover mediums for the purpose of producing anaerobiosis, probably acts by reducing the surface tension rather than by excluding oxygen.

\title{
EFFECT OF FOREIGN PROTEIN ON THE KIDNEY.
}

\author{
By E. T. Bell and T. B. Hartzell.
}

(From the Department of Pathology, University of Minnesota.)

$\mathrm{I}^{\mathrm{T}}$ T IS known that some persons develop symptoms of anaphylactic character after eating certain foods, of which shellfish, egg-white, and certain fruits are familiar examples. It is believed by many that asthma, urticaria and hay-fever have their origin largely in a condition of hypersensitiveness to special foreign proteins. The symptoms produced when a foreign protein is introduced into a sensitized animal are usually referred to a hypothetical substance-"anaphylatoxin"-formed by the action of antibody on antigen. There are reports in the literature to the effect that anatomic changes in the tissues occur in acute anaphylaxis, and Longcope $^{1}$ has advanced the theory that repeated anaphylactic shock may be re-

'Jour. Exper. Med., 1913, 18, p. 678; 1915,22 ,
p. 793; Tians. Assn. Am. Phys., 1914, 29, p. 19. spcnsible for such chronic diseases as nephritis. By a series of animal experiments Longcope believes that he has shown that repeated nonfatal poisoning with foreign protein is responsible for some cases of chronic nephritis, cirrhosis of the liver, and chronic myocarditis. Our interest in the etiology of chronic nephritis led us to repeat some of his experiments.

\section{Anatomic Changes in Acute Ana- PHYLAXIS.}

Gay and Southard ${ }^{2}$ described hemorrhages and areas of fatty degeneration in guinea-pigs that died in acute anaphylactic shock and in those killed subsequent to severe shock. The hemorrhages were widely distributed, but

${ }^{2}$ Jour. Med. Research, 1907, 16, p. 143; 1908, 18 , p. 17 . 